\title{
System Identification Algorithm Analysis of Acupuncture Effect on Mean Blood Flux of Contralateral Hegu Acupoint
}

\author{
Guangjun Wang, ${ }^{1}$ Jianguo Han, ${ }^{2}$ Gerhard Litscher, ${ }^{1,3}$ and Weibo Zhang1 \\ ${ }^{1}$ Department of Biomedical Engineering, Institute of Acupuncture and Moxibustion, China Academy of Chinese Medical Sciences, \\ Beijing 100700, China \\ ${ }^{2}$ Institute of Information Science and Technology, Beijing University of Chemical Technology, Beijing 100029, China \\ ${ }^{3}$ Stronach Research Unit for Complementary and Integrative Laser Medicine, Research Unit of Biomedical Engineering in \\ Anesthesia and Intensive Care Medicine, and TCM Research Center Graz, Medical University of Graz, Auenbruggerplatz 29, \\ 8036 Graz, Austria
}

Correspondence should be addressed to Gerhard Litscher, gerhard.litscher@medunigraz.at

Received 11 March 2012; Accepted 21 March 2012

Academic Editor: Lu Wang

Copyright ( 92012 Guangjun Wang et al. This is an open access article distributed under the Creative Commons Attribution License, which permits unrestricted use, distribution, and reproduction in any medium, provided the original work is properly cited.

Background. Acupoints (belonging to 12 meridians) which have the same names are symmetrically distributed on the body. It has been proved that acupoints have certain biological specificities different from the normal parts of the body. However, there is little evidence that acupoints which have the same name and are located bilaterally and symmetrically have lateralized specificity. Thus, researching the lateralized specificity and the relationship between left-side and right-side acupuncture is of special importance. Methodology and Principal Findings. The mean blood flux (MBF) in both Hegu acupoints was measured by Moor full-field laser perfusion imager. With the method of system identification algorithm, the output distribution in different groups was acquired, based on different acupoint stimulation and standard signal input. It is demonstrated that after stimulation of the right Hegu acupoint by needle, the output value of MBF in contralateral Hegu acupoint was strongly amplified, while after acupuncturing the left Hegu acupoint, the output value of MBF in either side Hegu acupoint was amplified moderately. Conclusions and Significance. This paper indicates that the Hegu acupoint has lateralized specificity. After stimulating the ipsilateral Hegu acupoint, symmetry breaking will be produced in contrast to contralateral Hegu acupoint stimulation.

\section{Introduction}

Acupuncture has been widely used to reduce some symptoms or to treat diseases in clinical practice for at least 2000 years [1]. During the past 30 years, a large number of studies focused on the antinociception mechanism of acupuncture, which made it more acceptable to clinical practice and mechanism research. According to the principles of Unschuld [2], acupuncture effects might be related to the appropriate acupoints during the treatment. However, previous studies have indicated that electroacupuncture (EA) is involved in modifying a variety of brain functions and in promoting the release of endogenous opioid peptides, which might be responsible for its analgesic effects in the whole body $[3,4]$. It means that acupuncture specificity contributes a little to its effect. What is more, many researchers firmly believe that placebo effect may be the best explanation for acupuncture $[5,6]$.

On the other hand, specificity of acupuncture points seems to be confirmed by the evidence from neuroimaging studies $[7,8]$. It has been shown that acupuncture at different acupoints induced differential hemodynamic neural responses in some brain areas [9]. In contrast, there is little evidence to discern the differences of acupoints which have the same name and are located bilaterally and symmetrically. Goldman et al. reported significant analgesic effects of 
ipsilateral but not contralateral acupoints in a mouse model of inflammatory pain [10]. Somers and Clemente [11] obtained opposite results: transcutaneous electric nerve stimulation on the contra- but not ipsilateral side of neuropathic pain resulted in antinociceptive effects in rats. Furthermore, a study [12] indicated that although the anti-nociceptive effect of both contralateral and ipsilateral EA was definitely confirmed, lesions of the rostral anterior cingulated cortex completely abolished the anti-nociceptive effects of contrabut not ipsilateral EA. These studies intensively suggested that there might be a difference between ipsilateral acupuncture and contralateral acupuncture.

In our lab in 1997, Zhang WB measured the transcutaneous $\mathrm{CO}_{2}$ emission on left and right 24 source acupoints and calculated the correlations between the points. It showed a significantly higher correlative coefficient ( 0.814$)$ between the left and right same name acupoints than the correlative coefficient between general acupoints (0.379) [13]. Our recent studies have also shown that thermostimulation could result in an increase of blood perfusion not only in the local area [14] but also in the same area on the contralateral side [15]. This phenomenon can be observed both in the upper limb [15] and lower limb [16]. However, the same stimulation has no effect on periumbilicus area [14], which indicated that there might be intrinsic and symmetrical correlation between contra- and ipsilateral parts. This view was supported by Kubo et al. [17]. Their work indicated that after acupuncture or thermostimulation in the ipsilateral side, the blood volume increased gradually in contralateral Achilles tendon, and the amount of increase in blood volume of the nontreated tendon (contralateral side) was significantly correlated to that of the treated tendon (ipsilateral side) during the last phase of recovery period. Recently we reported that when either side Hegu acupoint (LI4) was stimulated, there was an increase in mean blood flux at LI4 of the contralateral side. However, the intrinsic correlation between contra- and ipsilateral LI4 is not clear. The purpose of this study is to investigate the correlation of bilateral LI4 through system identification algorithm analysis.

\section{Methods}

2.1. Ethics Statement. This study was reviewed and approved by the Institutional Review Board at the Institute of Acupuncture and Moxibustion, China Academy of Chinese Medical Sciences. Each participant read and signed an informed consent form.

2.2. Subjects. One hundred and twenty (120) healthy volunteers were recruited in this study (as shown in Figure 1; for demographic data see Table 1). All subjects were students from the China Academy of Chinese Medical Sciences and Beijing University of Traditional Chinese Medicine. All subjects had no history of diseases and had not taken any medicine in the past six months before the experiment. Each subject had an adequate understanding of the procedure and purpose of this study.
TABLE 1: Subjects' demographic data.

\begin{tabular}{lccc}
\hline Group & $n$ & $\begin{array}{c}\text { Gender } \\
\text { (female/male) }\end{array}$ & $\begin{array}{c}\text { Age (years, } \\
\text { mean } \pm \text { SD) }\end{array}$ \\
\hline AL group & 40 & $34 / 6$ & $25.34 \pm 1.77$ \\
AR group & 40 & $25 / 15$ & $25.85 \pm 1.24$ \\
Control group & 40 & $34 / 6$ & $25.33 \pm 1.69$ \\
\hline
\end{tabular}

AL: acupuncture left Hegu point; AR: acupuncture right Hegu point.

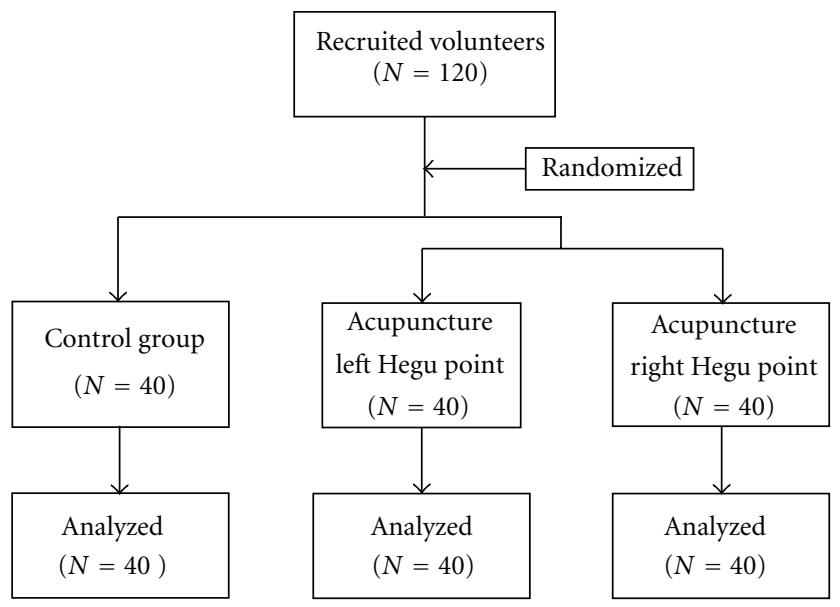

FIgURE 1: Flow diagram of participants in the study.

\subsection{Procedures}

2.3.1. Protocol for Mean Blood Flux Measurement. Before arrival to the laboratory, subjects were placed in a temperaturecontrolled room $\left(24-26^{\circ} \mathrm{C}\right)$ as a resting state for 60 minutes. Measurements of skin blood perfusion were carried out using Moor full-field laser perfusion imager (moor FLPI, Moor Instruments Ltd, UK). Before recording, both hands were immobilized with a cylindrical object to ensure positioning. The measurement parameters were as follows: high resolution $/ 250$ frames; number of images $=10$; exposure time $=$ $8.3 \mathrm{~ms}$; time interval $=10 \mathrm{~s}$. Measurements were carried out every 30 minutes over a total of 180 minutes. During the experiment, the laboratory room was kept in dark light condition, and the protocol for measurement operation was abided strictly. The measurement process is illustrated in Figure 2 .

2.3.2. Acupuncture Protocol. For acupuncture, a small acupuncture needle, $0.25 \times 25 \mathrm{~mm}$ (100112, Zhen Huan), was gently inserted in a depth of $15 \mathrm{~mm}$ in the LI4. The position of LI4 was confirmed according to the previous studies [18, 19]. The needle was slowly rotated every $5 \mathrm{~min}$ for a total of 30 min during an acupuncture session in order to maintain the soreness and numbness sensation of De-Qi [10]. The acupuncture procedure is illustrated in Figure 2. In the left acupuncture group (Left Acup.), just left LI4 was acupunctured whereas in the right acupuncture group (Right Acup.), right LI4 was stimulated. In the control group (No Acup.), all subjects maintained still, without any intervention. 


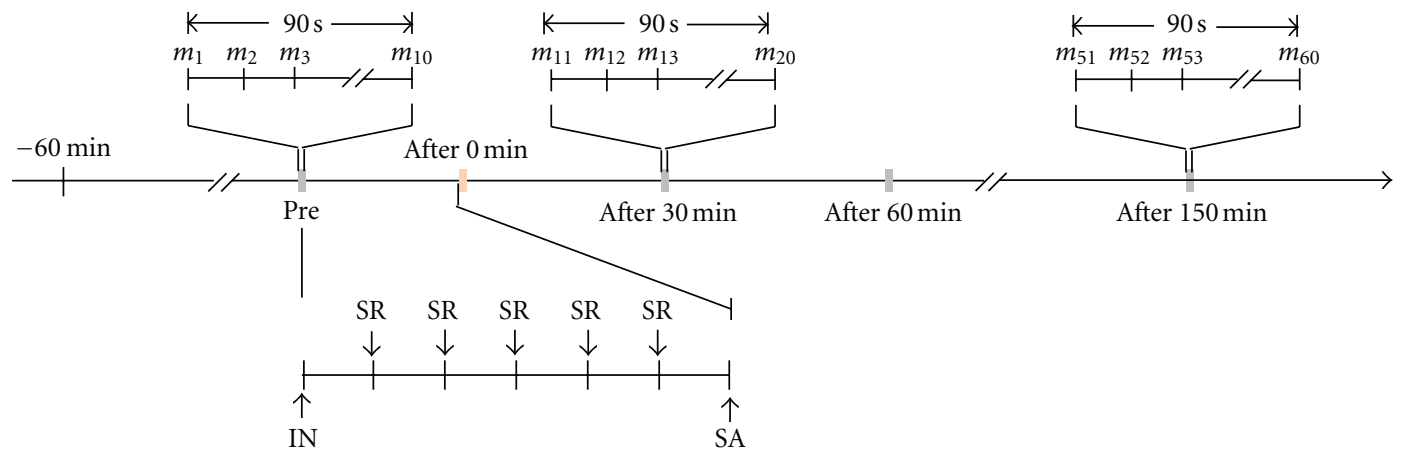

FIGURE 2: Procedure of acupuncture and mean blood flux measurement. Pre: pre acupuncture; Post, post acupuncture; IN: insert needle; SA: stop acupuncture; SR: slowly rotate the needle every five minutes; $m_{i}(i=1,2,3, \ldots, 60)$ : mean blood flux of Hegu acupoint at a specific time point.

2.3.3. Image Analysis Protocol. The Mean Blood Flux (MBF) of LI4 on both hands (left side abbreviated as L, right side abbreviated as R) was measured (Figure 3). In order to exclude the nonspecific effect of acupuncture practice, a total of 10 data from the post-0-minute phase were excluded from the final analysis (Figure 2). We symbolized left acupuncture group, right acupuncture group, and control group as $\mathrm{A}, \mathrm{B}$, and $C$, respectively. So for every person, there are 60 pairs of data acquired. For every group, there are 2400 pairs of data acquired. We denoted the mapping relationship of these data pairs, namely, mapping relationship from $\{A L\}$ to $\{A R\}$, from $\{B L\}$ to $\{B R\}$, from $\{C L\}$ to $\{C R\}$ as $\{A L\} \rightarrow\{A R\}$, $\{\mathrm{BL}\} \rightarrow\{\mathrm{BR}\}$ and $\{\mathrm{CL}\} \rightarrow\{\mathrm{CR}\}$, and summarized as $\left\{\begin{array}{c}\mathrm{A} \\ \mathrm{BL} \\ \mathrm{C}\end{array}\right\} \rightarrow\left\{\begin{array}{c}\mathrm{A} \\ \mathrm{BR} \\ \mathrm{C}\end{array}\right\}$.

2.3.4. System Identification Algorithm. Under the condition of $\left\{\begin{array}{c}\mathrm{A} \\ \mathrm{BL} \\ \mathrm{C}\end{array}\right\} \rightarrow\left\{\begin{array}{c}\mathrm{A} \\ \mathrm{BR} \\ \mathrm{C}\end{array}\right\}$, totally $2400 \times 3$ pairs of data were acquired and symbolized as $f_{\mathrm{AL}}(k), f_{\mathrm{BL}}(k), f_{\mathrm{CL}}(k), f_{\mathrm{AR}}(k)$, $f_{\mathrm{BR}}(k), f_{\mathrm{CR}}(k), k=1,2, \ldots, 2400$, where A represents left acupuncture group; B represents right acupuncture group; $\mathrm{C}$ represents control group; L represents left Hegu acupoint; $\mathrm{R}$ represents right Hegu acupoint; $k=1,2, \ldots, 2400$. Then, we determined $f_{\mathrm{AL}}(k), f_{\mathrm{BL}}(k)$ and $f_{\mathrm{CL}}(k)$, as input and $f_{\mathrm{AR}}(k)$, $f_{\mathrm{BR}}(k)$, and $f_{\mathrm{CR}}(k)$ as output, respectively. System identification algorithm was performed in the Matlab software (Version: 6.5). The flow diagram of system identification algorithm is shown in Figure 4.

\section{Results}

3.1. Mapping Model. Three mathematical models were obtained as follows by executing system identification algorithm, which reflects the correlation of bilateral Hegu acupoints under different intervention conditions.

Model 1:

$$
\begin{aligned}
f_{\mathrm{AL}}(k)= & a_{1} f_{\mathrm{AL}}(k-1)+b_{1}\left[f_{\mathrm{AL}}(k-1)\right]^{0.3} \\
& +c_{1} f_{\mathrm{AR}}(k)+d_{1}\left[f_{\mathrm{AR}}(k)\right]^{0.3} .
\end{aligned}
$$

Model 2:

$$
\begin{gathered}
f_{\mathrm{BL}}(k)=a_{2} f_{\mathrm{BL}}(k-1)+b_{2}\left[f_{\mathrm{BL}}(k-1)\right]^{0.3} \\
+c_{2} f_{\mathrm{BR}}(k)+d_{2}\left[f_{\mathrm{BR}}(k)\right]^{0.3} .
\end{gathered}
$$

Model 3:

$$
\begin{aligned}
f_{\mathrm{CL}}(k)= & a_{3} f_{\mathrm{CL}}(k-1)+b_{3}\left[f_{\mathrm{CL}}(k-1)\right]^{0.3} \\
& +c_{3} f_{\mathrm{CR}}(k)+d_{3}\left[f_{\mathrm{BR}}(k)\right]^{0.3} .
\end{aligned}
$$

Using the values (measured on the left side) of $f_{\mathrm{AL}}(k)$, $f_{\mathrm{BL}}(k)$, and $f_{\mathrm{CL}}(k)$ as input variables of models (1), (2), and (3), the estimated value (mapping value) of the right Hegu acupoint can be obtained with help of the models, symbolized as $f_{\mathrm{AR}}{ }^{*}(k), f_{\mathrm{BR}}{ }^{*}(k)$ and $f_{\mathrm{CR}}{ }^{*}(k)$, following the mapping relationship $f_{\mathrm{AL}}(k) \rightarrow f_{\mathrm{AR}}^{*}(k), f_{\mathrm{BL}}(k) \rightarrow f_{\mathrm{BR}}^{*}(k)$, and $f_{\mathrm{CL}}(k) \rightarrow f_{\mathrm{CR}}{ }^{*}(k)$.

3.2. Error Evaluation and Signal-Noise Ratio. The errors between "true" value and their estimated values (symbolized as $\left.d_{i}(k)\right)$ are defined as

$$
\begin{aligned}
& d_{\mathrm{A}}(k)=f_{\mathrm{AR}}{ }^{*}(k)-f_{\mathrm{AR}}(k), \quad k=1,2, \ldots, 2400, \\
& d_{\mathrm{B}}(k)=f_{\mathrm{BR}}{ }^{*}(k)-f_{\mathrm{BR}}(k), \quad k=1,2, \ldots, 2400, \\
& d_{\mathrm{C}}(k)=f_{\mathrm{CR}}{ }^{*}(k)-f_{\mathrm{CR}}(k), \quad k=1,2, \ldots, 2400 .
\end{aligned}
$$

Then we defined the signal-noise ratio $\operatorname{sn}_{i}(i=A, B, C)$ as

$$
\begin{aligned}
& \operatorname{sn}_{\mathrm{A}}=\frac{\left\{\sqrt{\sum_{k=1}^{2400}\left[f_{\mathrm{AR}}{ }^{*}(k)\right]^{2}} / \sqrt{\sum_{k=1}^{2400}\left[d_{\mathrm{A}}(k)\right]^{2}}\right\}}{2400}, \\
& \operatorname{sn}_{\mathrm{B}}=\frac{\left\{\sqrt{\sum_{k=1}^{2400}\left[f_{\mathrm{BR}}^{*}(k)\right]^{2}} / \sqrt{\sum_{k=1}^{2400}\left[d_{\mathrm{B}}(k)\right]^{2}}\right\}}{2400}, \\
& \operatorname{sn}_{\mathrm{C}}=\frac{\left\{\sqrt{\sum_{k=1}^{2400}\left[f_{\mathrm{CR}} *(k)\right]^{2}} / \sqrt{\sum_{k=1}^{2400}\left[d_{\mathrm{C}}(k)\right]^{2}}\right\}}{2400} .
\end{aligned}
$$




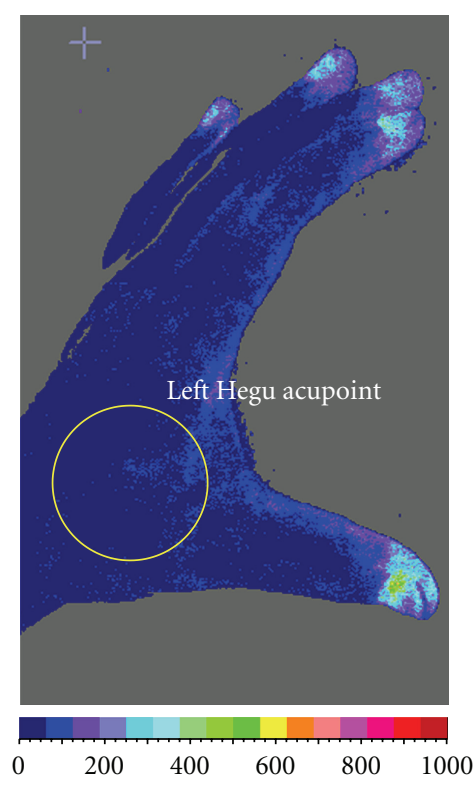

(a)

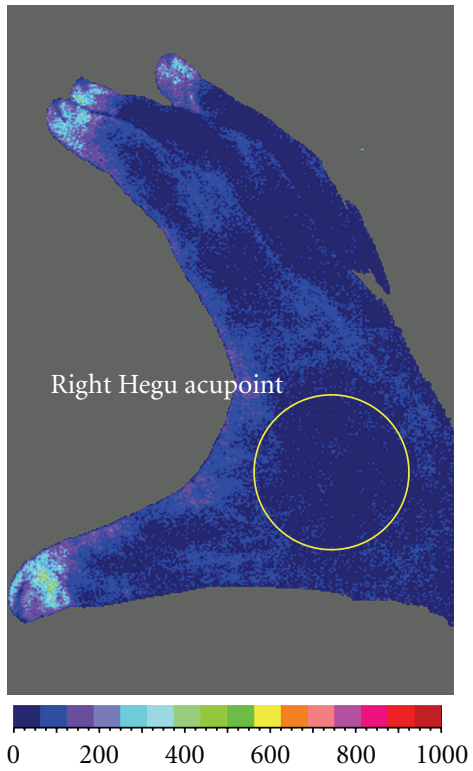

(b)

Figure 3: Confirmation of Hegu acupoint. (a) left hand, (b) right hand.

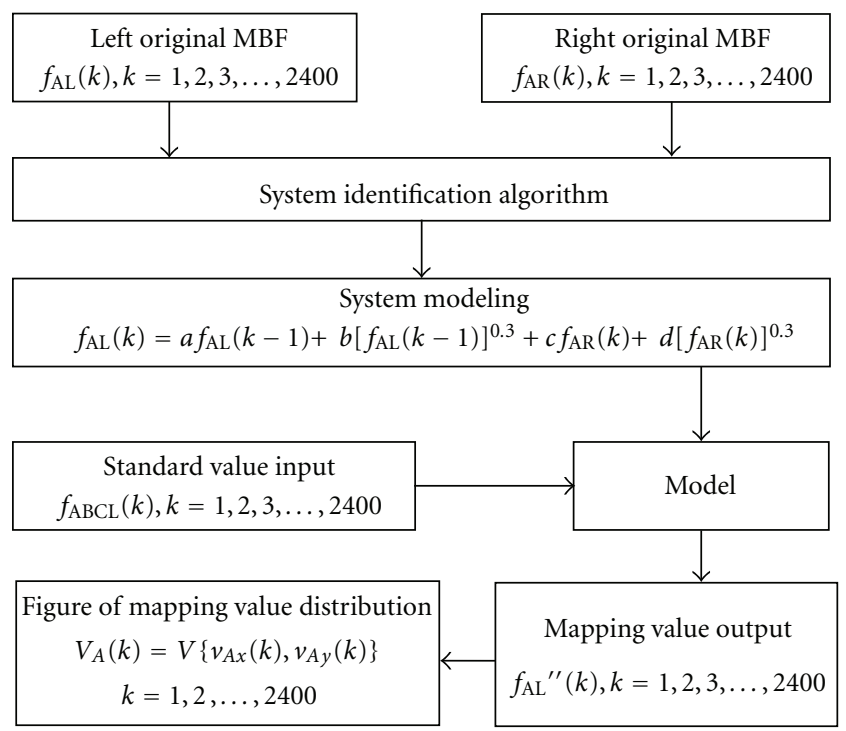

FIgURE 4: Flow diagram of system identification algorithm.

3.3. Standard Signal Input. Standard signal value series used as common model-input value series denoted as $f_{\mathrm{ABCL}}(k)$ are mathematically calculated as

$$
\begin{aligned}
& f_{\mathrm{ABCL}}(k) \\
& \begin{aligned}
=10 \times\left\{\sin \left(\frac{k}{100}\right)+\sin \left(\frac{k}{80}\right)+\sin \left(\frac{k}{60}\right)\right. \\
\quad+\sin \left(\frac{k}{40}\right)+\cos \left(\frac{k}{90}\right)+\cos \left(\frac{k}{70}\right)+ \\
\left.\quad+\cos \left(\frac{k}{50}\right)+\cos \left(\frac{k}{30}\right)\right\} \quad k=1,2, \ldots, 2400 .
\end{aligned}
\end{aligned}
$$

The standard signal input is to maintain the complexity and stability. If $f_{\mathrm{AL}}(k), f_{\mathrm{BL}}(k)$, and $f_{\mathrm{CL}}(k)$ were all replaced by $f_{\mathrm{ABCL}}(k)$, the output $f_{\mathrm{AL}}{ }^{\prime \prime}(k), f_{\mathrm{BL}}{ }^{\prime \prime}(k)$, and $f_{\mathrm{CL}}{ }^{\prime \prime}(k)$ will be produced with help of the models, instead of $f_{\mathrm{AR}}{ }^{*}(k)$, $f_{\mathrm{BR}}{ }^{*}(k)$, and $f_{\mathrm{CR}}{ }^{*}(k)$, respectively. Thus the mapping relationship will change into $f_{\mathrm{ABCL}}(k) \rightarrow f_{\mathrm{AL}}{ }^{\prime \prime}(k), f_{\mathrm{ABCL}}(k) \rightarrow$ $f_{\mathrm{BL}}{ }^{\prime \prime}(k), f_{\mathrm{ABCL}}(k) \rightarrow f_{\mathrm{CL}}{ }^{\prime \prime}(k)$.

3.4. Determination of Characteristic Vectors. In order to set up model output characteristic vectors to describe the different mapping results of different interventions, we define one subvector of the model output characteristic vector as

$$
\begin{array}{ll}
v_{\mathrm{AL} y}(k)=f_{\mathrm{AL}}{ }^{\prime \prime}(k), & k=1,2,3, \ldots, 2400, \\
v_{\mathrm{BL} y}(k)=f_{\mathrm{BL}}{ }^{\prime \prime}(k), & k=1,2,3, \ldots, 2400, \\
v_{\mathrm{CL} y}(k)=f_{\mathrm{CL}}{ }^{\prime \prime}(k), & k=1,2,3, \ldots, 2400 .
\end{array}
$$

The other subvector of the model output characteristic vector was determined as

$$
\begin{array}{ll}
v_{\mathrm{A} x}(k)=\mathrm{sn}_{\mathrm{A}}+d_{\mathrm{A}}(k), & k=1,2, \ldots, 2400, \\
v_{\mathrm{B} x}(k)=\mathrm{sn}_{\mathrm{B}}+d_{\mathrm{B}}(k), & k=1,2, \ldots, 2400, \\
v_{\mathrm{C} x}(k)=\mathrm{sn}_{\mathrm{C}}+d_{\mathrm{C}}(k), & k=1,2, \ldots, 2400 .
\end{array}
$$

Then the 2-dimensional diagram of mapping value distribution was produced. In the left acupuncture group, the distribution of output was determined as

$$
V_{\mathrm{A}}(k)=V\left\{v_{\mathrm{A} x}(k), v_{\mathrm{A} y}(k)\right\}, \quad k=1,2, \ldots, 2400 .
$$

In the right acupuncture group, the distribution of output was determined as

$$
V_{\mathrm{B}}(k)=V\left\{v_{\mathrm{B} x}(k), v_{\mathrm{B} y}(k)\right\}, \quad k=1,2, \ldots, 2400 .
$$


TABLE 2: Mapping value distribution center and signal-noise ratio in different groups.

\begin{tabular}{lcc}
\hline $\begin{array}{l}\text { Original } \\
\text { input/output }\end{array}$ & $\begin{array}{c}\text { Intervention } \\
\text { method }\end{array}$ & $\begin{array}{c}\text { Distribution } \\
\text { center (PU) }\end{array}$ \\
\hline$f_{\mathrm{AL}}(k) \rightarrow f_{\mathrm{AR}}(k)$ & Acup. Left & 255.41 \\
$f_{\mathrm{BL}}(k) \rightarrow f_{\mathrm{BR}}(k)$ & Acup. Right & 591.01 \\
$f_{\mathrm{CL}}(k) \rightarrow f_{\mathrm{CR}}(k)$ & No acup. & 71.58 \\
$f_{\mathrm{AR}}(k) \rightarrow f_{\mathrm{AL}}(k)$ & Acup. Left & 222.32 \\
$f_{\mathrm{BR}}(k) \rightarrow f_{\mathrm{BL}}(k)$ & Acup. Right & 965.81 \\
$f_{\mathrm{CR}}(k) \rightarrow f_{\mathrm{CL}}(k)$ & No acup. & 96.33 \\
\hline
\end{tabular}

In the control group, the distribution of output was determined as

$$
V_{\mathrm{C}}(k)=V\left\{v_{\mathrm{C} x}(k), v_{\mathrm{C} y}(k)\right\}, \quad k=1,2, \ldots, 2400 .
$$

The output distribution is shown in Figure 5(b). To exclude the possibility that these results were lateralized to one side, we defined the original $f_{\mathrm{AR}}(k), f_{\mathrm{BR}}(k), f_{\mathrm{CR}}(k)$ as input, $f_{\mathrm{AL}}(k), f_{\mathrm{BL}}(k), f_{\mathrm{CL}}(k)$ as output, respectively, summarized as $\left\{\begin{array}{c}\mathrm{A} \\ \mathrm{BR} \\ \mathrm{C}\end{array}\right\} \rightarrow\left\{\begin{array}{c}\mathrm{A} \\ \mathrm{BL} \\ \mathrm{C}\end{array}\right\}$. Then the system identification algorithm was carried out with MATLAB software again, and the other 3 models were produced. When the same standard signals were input into the different models, the distribution of output was produced (Figure 5(a)). Mapping value distribution center and signal-noise ratio in different groups were shown in Table 2.

From Figure 5, we can find that stimulation of right LI4 has the strong amplification effect on blood perfusion in left LI4, and this strong amplification effect is independent of the original input and output selection in the system identification algorithm analysis. In contrast, acupuncture at left LI4 just produces moderate amplification effects on blood perfusion in right LI4, and this moderate amplification is independent of the original input and output selection too. There is no amplification effect produced in the control group. These results indicated that after acupuncture, the amount of amplification effect on blood perfusion in contralateral side was just related to which lateral acupoint was acupunctured. $i=\mathrm{A}, \mathrm{B}, \mathrm{C}, k=1,2,3, \ldots, 2400$. (A) under the condition of $\left\{\begin{array}{c}\mathrm{A} \\ \mathrm{BR} \\ \mathrm{C}\end{array}\right\} \rightarrow\left\{\begin{array}{c}\mathrm{A} \\ \mathrm{BL} \\ \mathrm{C}\end{array}\right\}$. (B) under the condition of $\left\{\begin{array}{c}\mathrm{A} \\ \mathrm{BR} \\ \mathrm{C}\end{array}\right\} \rightarrow\left\{\begin{array}{c}\mathrm{A} \\ \mathrm{BL} \\ \mathrm{C}\end{array}\right\}$. A, acupuncture left Hegu acupoint; $\mathrm{B}$, acupuncture right Hegu acupoint; $\mathrm{C}$, no acupuncture.

\section{Discussion}

"In physics, symmetry means uniformity or invariance" [20], in other words, "the existence of different viewpoints from which the system appears the same" [21]. In Traditional Chinese Medicine (TCM), the principle is to maintain the body balance. Under the guidance of TCM theory, clinical practice is always in the pursuit of balance and symmetry. For example, according to the Neijing theory, if someone has disease in the left body, the treatment point is usually selected in the right side, and vice versa. However, "increasing levels of broken symmetry in many-body systems correlates with increasing complexity and functional specialization" [20]. In acupuncture theory, the symmetry breaking means there are differences between two meridians or two acupoints which have the same name and are located bilaterally and symmetrically on the body.

Recently, a system review analyzed the contralateral and ipsilateral acupuncture effect on poststroke hemiplegic patients [22]. Although this system review and meta-analysis could not come to a definitive conclusion, it indicates the importance of distinction between contralateral and ipsilateral acupuncture. According to traditional acupuncture theory, if we stimulate LI4 on one side, the function of the large intestine meridian (LI) located on the other side might also be activated. As a result, the running of Qi and blood which flow in both LI meridians were changed. So the basis of contra- or ipsilateral acupuncture is the specificity of acupoints which have the same name.

But up to now, it is difficult to evaluate the activation of acupoints, and, as a result, it is also difficult to analyse the specificity of acupoints after meridians are stimulated. Recently, more and more attention has been focused on the relationship of acupuncture and circulation [23-25]. In TCM theory, one of the definitive causes of acupuncture effect is the special sensation in local acupoints after stimulation, which might be related to the blood perfusion changes in acupoints or meridians [18]. According to the previous study, the mean blood flux (MBF) was larger at the acupoints than in their surrounding tissues, which indicates that the $\mathrm{MBF}$ can be used as an index for discriminating differences in the microcirculatory conditions between acupoints and their surrounding tissues [26]. It has also been shown that acupuncture can not only increase general circulation [27] and circulation in specific organs [28] but also change the skin microcirculation as well $[19,24,29,30]$. When an acupoint was stimulated adequately, the blood perfusion of this point continued to increase whereas the blood perfusion of nonacupoint only changed slightly by the same acupuncture stimulation [31]. These results indicated that the blood perfusion in acupoints can be recommended as candidate for acupuncture effect evaluation.

Our previous study has shown that ipsilateral acupoint stimulation could result in an increase of blood perfusion in contralateral side. But the lateralized characteristic is still not clear. This study indicated that the stimulation effect was different in different intervention groups. After stimulation of right LI4, the amplification effect on blood perfusion in contralateral is better than that in other two groups. It means under resting condition, the mean blood flux in both Hegu acupoints is symmetrical; after stimulating either side Hegu acupoint, this symmetry is broken. As a result, the MBF in the contralateral acupoint was amplified. But this amplification effect is different in different groups, which might be another phenomenon of symmetry breaking on a high level.

According to our previous study [32], under anesthesia condition, thermostimulation has no effect on the blood perfusion in the contralateral side foot. These results indicated that this asymmetry phenomenon was strengthened by the 

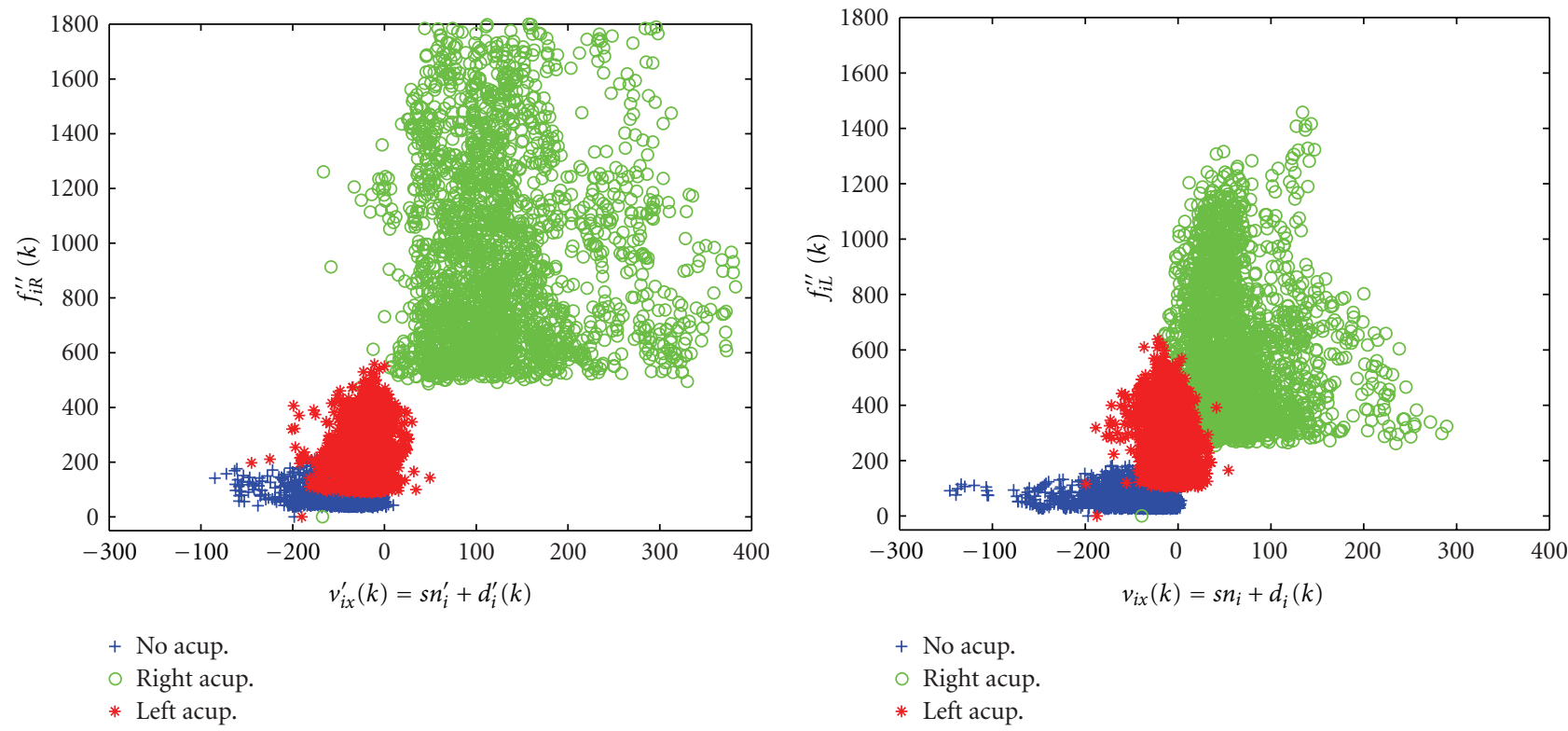

(a)

(b)

FIGURE 5: Mapping value distribution of standard input in different models.

anesthesia. In other words, synchronous changes of bilateral blood perfusion might be related to the wakefulness status. Although it is difficult to explore the reasons, we think it might be related to the asymmetry of brain.

\section{Conflict of Interests}

The authors declare that they have no conflict of interests.

\section{Acknowledgments}

This research was supported by the National Natural Science Foundation of China (Grant no. 81001553) and a SinoAustrian cooperating project on high-tech acupuncture. The research activities at the TCM Research Center Graz are partly supported by the German Academy of Acupuncture (President Dr. Gerhard Opitz). Professor G. Litscher is also a Visiting Professor at the Institute of Acupuncture and Moxibustion at the China Academy of Chinese Medical Sciences.

\section{References}

[1] G. D. Lu and J. Needham, Celestial Lancets: A History and Rationale of Acupuncture and Moxa, Cambridge University Press, New York, NY, USA, 1980.

[2] P. U. Unschuld, Huang Di Nei Jing Su Wen: Nature, Knowledge, Imagery in an Ancient Chinese Medical Text, with an Appendix, The Doctrine of The Five Periods and Six Qi in The Huang Di Nei Jing Su Wen, University of California Press, Berkeley, Calif, USA, 2003.

[3] Z. Q. Zhao, "Neural mechanism underlying acupuncture analgesia," Progress in Neurobiology, vol. 85, no. 4, pp. 355-375, 2008.
[4] J. S. Han, "Acupuncture: neuropeptide release produced by electrical stimulation of different frequencies," Trends in Neurosciences, vol. 26, no. 1, pp. 17-22, 2003.

[5] M. E. Wechsler, J. M. Kelley, I. O. Boyd et al., "Active albuterol or placebo, sham acupuncture, or no intervention in asthma," The New England Journal of Medicine, vol. 365, no. 2, pp. 119126, 2011.

[6] Y. C. Park, W. Kang, S. M. Choi, and C. G. Son, "Evaluation of manual acupuncture at classical and nondefined points for treatment of functional dyspepsia: a randomized-controlled trial," Journal of Alternative and Complementary Medicine, vol. 15, no. 8, pp. 879-884, 2009.

[7] L. Li, H. Liu, Y. Z. Li et al., "The human brain response to acupuncture on same-meridian acupoints: evidence from an fMRI study," Journal of Alternative and Complementary Medicine, vol. 14, no. 6, pp. 673-678, 2008.

[8] L. J. Bai, H. Yan, L. L. Li et al., "Neural specificity of acupuncture stimulation at pericardium 6: evidence from an fMRI study," Journal of Magnetic Resonance Imaging, vol. 31, no. 1, pp. 71-77, 2010.

[9] Y. S. Ren, L. J. Bai, Y. Y. Feng, J. Tian, and K. C. Li, "Investigation of acupoint specificity by functional connectivity analysis based on graph theory," Neuroscience Letters, vol. 482, no. 2, pp. 95-100, 2010.

[10] N. Goldman, M. Chen, T. Fujita et al., "Adenosine A1 receptors mediate local anti-nociceptive effects of acupuncture," Nature Neuroscience, vol. 13, no. 7, pp. 883-888, 2010.

[11] D. L. Somers and F. R. Clemente, "Transcutaneous electrical nerve stimulation for the management of neurophatic pain: the effects of frequency and electrode position on prevention of allodynia in a rat model of complex regional pain syndrome type II," Physical Therapy, vol. 86, no. 5, pp. 698-709, 2006.

[12] M. Yi, H. Zhang, L. Lao, G. G. Xing, and Y. Wan, "Anterior cingulate cortex is crucial for contra- but not ipsi-lateral electro-acupuncture in the formalin-induced inflammatory pain model of rats," Molecular Pain, vol. 7, no. 1, p. 61, 2011. 
[13] W. B. Zhang, "Cluster analysis to the correlativity of skin respiration of $\mathrm{CO}_{2}$ on acupoints of twelve meridians on human body," Journal of Biomathematics, vol. 12, no. 3, pp. 261-264, 1997.

[14] G. J. Wang, Y. Q. Zhang, R. H. Wang et al., "Experimental study on effect of electro-heated stone needle on local skin blood perfusion of dorsum hand," Tianjin Journal of Traditional Chinese Medicine, vol. 26, no. 5, pp. 382-384, 2009.

[15] G. J. Wang, Y. Q. Zhang, R. H. Wang et al., "The study of interaction based on the thermostimulation," Chinese Journal of Basic Medicine in Traditional Chinese Medicine, vol. 16, no. 9, pp. 803-811, 2010.

[16] Y. Q. Zhang, Y. L. Ding, Y. Y. Tian, T. Huang, W. B. Zhang, and G. J. Wang, "Change of blood perfusion on contra-lateral lower limb after electro-bian stone intervention," Jiangsu Journal of Traditional Chinese Medicine, vol. 42, no. 04, pp. 48-49, 2010.

[17] K. Kubo, H. Yajima, M. Takayama, T. Ikebukuro, H. Mizoguchi, and N. Takakura, "Changes in blood circulation of the contralateral achilles tendon during and after acupuncture and heating," International Journal of Sports Medicine, vol. 32, no. 10 , pp. $807-813,2011$.

[18] T. C. Kuo, C. W. Lin, and F. M. Ho, "The soreness and numbness effect of acupuncture on skin blood flow," American Journal of Chinese Medicine, vol. 32, no. 1, pp. 117-129, 2004.

[19] H. Hsiu, W. C. Hsu, B. H. Chen, and C. L. Hsu, "Differences in the microcirculatory effects of local skin surface contact pressure stimulation between acupoints and nonacupoints: possible relevance to acupressure," Physiological Measurement, vol. 31, no. 6, pp. 829-841, 2010.

[20] Q. F. Wu, Q. Zhang, B. Sun et al., " $1 \mathrm{H}$ NMR-based metabonomic study on the metabolic changes in the plasma of patients with functional dyspepsia and the effect of acupuncture," Journal of Pharmaceutical and Biomedical Analysis, vol. 51, no. 3, pp. 698-704, 2010.

[21] P. W. Anderson, “More is different," Science, vol. 177, no. 4047, pp. 393-396, 1972.

[22] M. K. Kim, T. Y. Choi, M. S. Lee, H. Lee, and C. H. Han, "Contralateral acupuncture versus ipsilateral acupuncturein the rehabilitation of post-stroke hemiplegic patients: a systematic review," BMC Complementary and Alternative Medicine, vol. 10, article 41, 2010.

[23] G. Litscher, L. Wang, E. Huber, and G. Nilsson, "Changed skin blood perfusion in the fingertip following acupuncture needle introduction as evaluated by laser doppler perfusion imaging," Lasers in Medical Science, vol. 17, no. 1, pp. 19-25, 2002.

[24] H. Hsiu, W. C. Hsu, S. L. Chang, C. L. Hsu, S. M. Huang, and Y. Y. W. Lin, "Microcirculatory effect of different skin contacting pressures around the blood pressure," Physiological Measurement, vol. 29, no. 12, pp. 1421-1434, 2008.

[25] G. Litscher, "Bioengineering assessment of acupuncture, part 2: monitoring of microcirculation," Critical Reviews in Biomedical Engineering, vol. 34, no. 4, pp. 273-293, 2006.

[26] H. Hsiu, S. M. Huang, P. T. Chao et al., "Microcirculatory characteristics of acupuncture points obtained by laser doppler flowmetry," Physiological Measurement, vol. 28, no. 10, pp. N77-N86, 2007.

[27] H. Niimi and H. S. Yuwono, "Asian traditional medicine: from molecular biology to organ circulation," Clinical Hemorheology and Microcirculation, vol. 23, no. 2-4, pp. 123-125, 2000.

[28] H. Tsuru and K. Kawakita, "Acupuncture on the blood flow of various organs measured simultaneously by colored microspheres in rats," Evidence-Based Complementary and Alternative Medicine, vol. 6, no. 1, pp. 77-83, 2009.
[29] H. Hsiu, W. C. Hsu, C. L. Hsu, M. Y. Jan, and Y. Y. Wang-Lin, "Effects of acupuncture at the hoku acupoint on the pulsatile laser doppler signal at the heartbeat frequency," Lasers in Medical Science, vol. 24, no. 4, pp. 553-560, 2009.

[30] M. L. Sandberg, M. K. Sandberg, and J. Dahl, "Blood flow changes in the trapezius muscle and overlying skin following transcutaneous electrical nerve stimulation," Physical Therapy, vol. 87, no. 8, pp. 1047-1055, 2007.

[31] T. C. Kuo, Z. S. Chen, C. H. Chen, F. M. Ho, C. W. Lin, and Y. J. Chen, "The physiological effect of DE QI during acupuncture," Journal of Health Science, vol. 50, no. 4, pp. 336342, 2004.

[32] G. J. Wang, Y. Q. Zhang, R. H. Wang, Y. Y. Tian, T. Huang, and W. B. Zhang, "The study of blood perfusion of foot skin in both lower extremity after injected with compound 48/80 in the right side," Shaanxi Journal of Traditional Chinese Medicine, vol. 31, no. 08, pp. 1070-1073, 2010. 


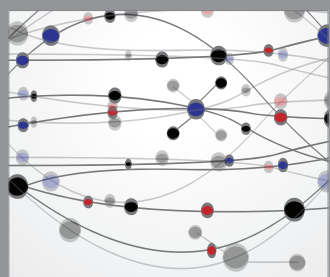

The Scientific World Journal
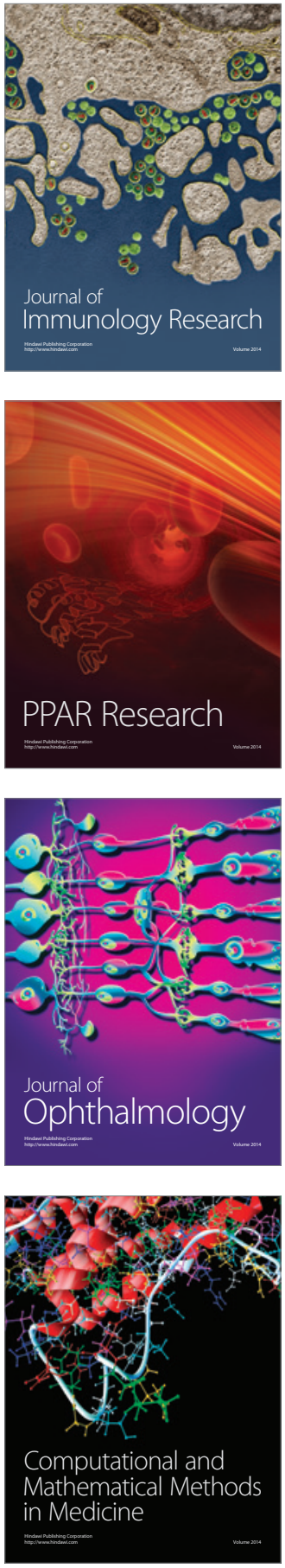

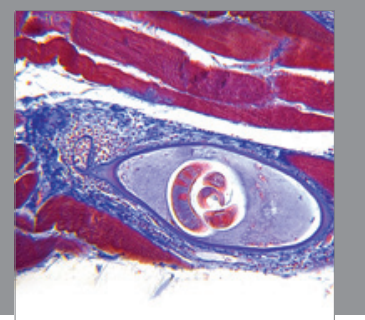

Gastroenterology

Research and Practice
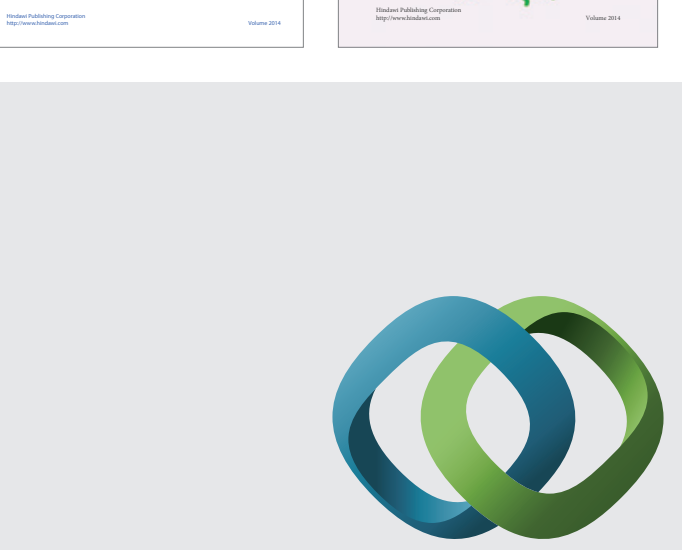

\section{Hindawi}

Submit your manuscripts at

http://www.hindawi.com
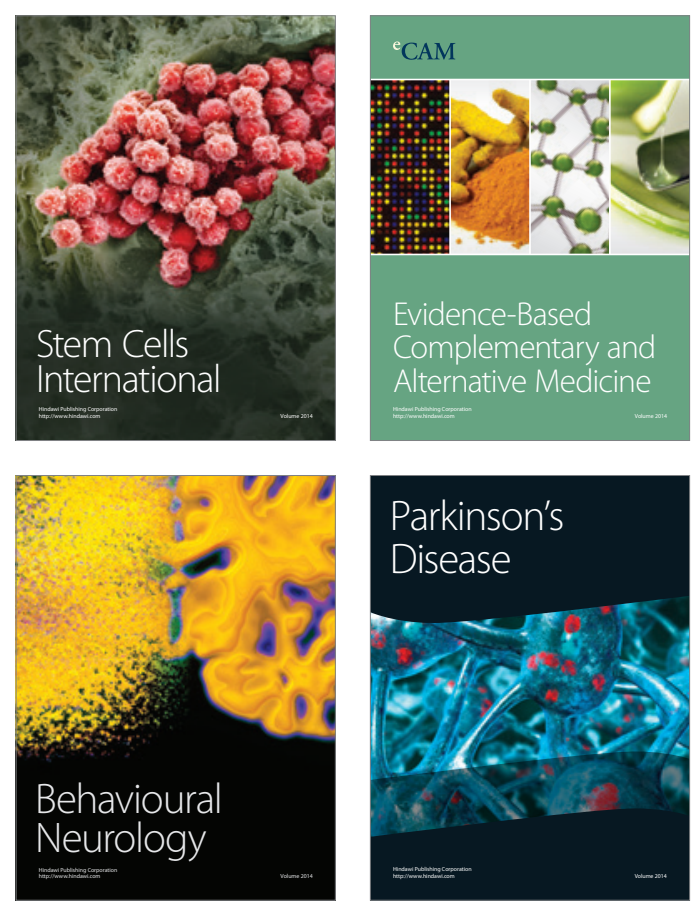

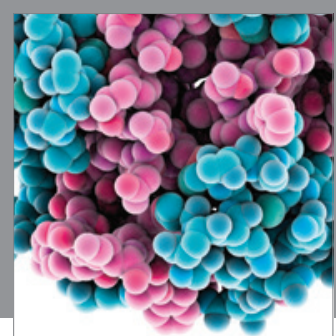

Journal of
Diabetes Research

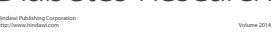

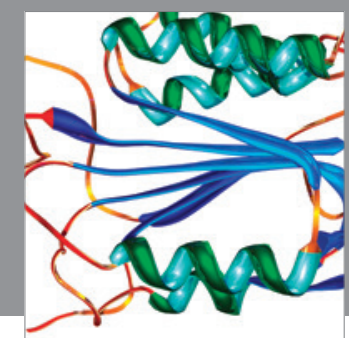

Disease Markers
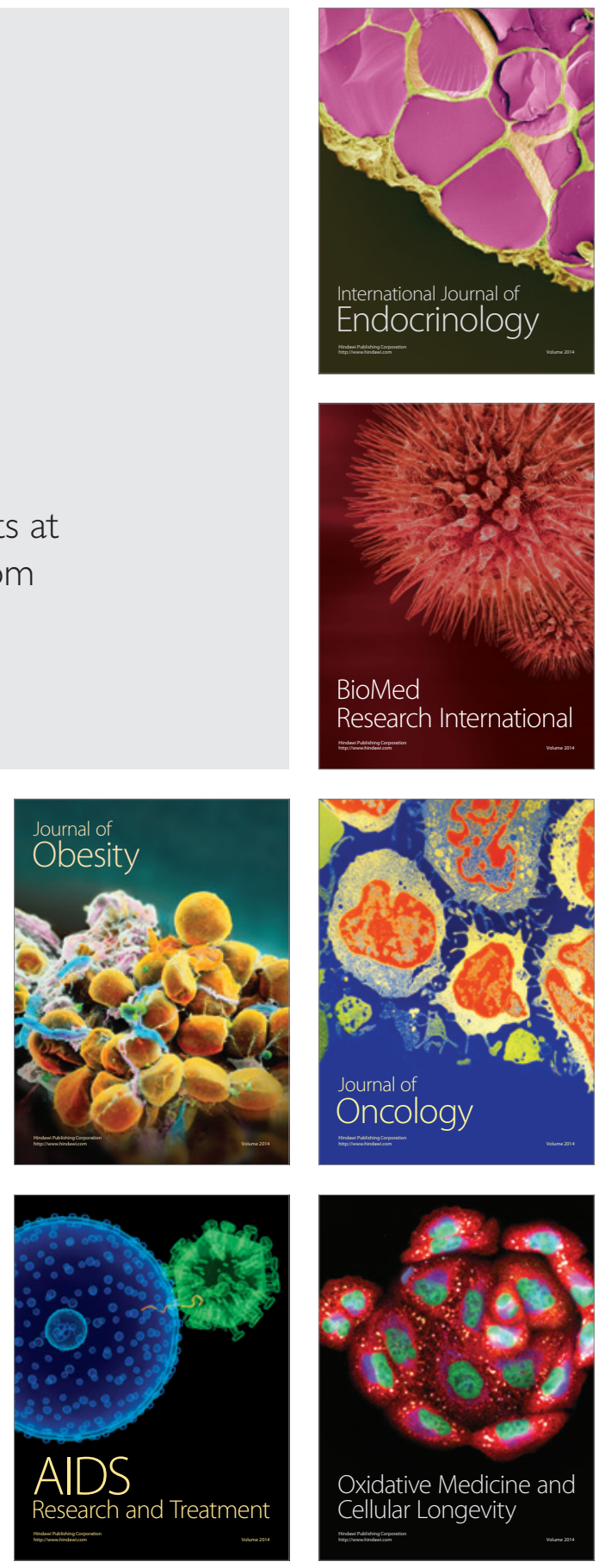